\title{
Mutant monocyte chemoattractant protein-1 protein (7ND) inhibits osteoclast differentiation and reduces oral squamous carcinoma cell bone invasion
}

\author{
SHUYU LUO ${ }^{1 *}$, CHUANXIANG ZHOU ${ }^{2 *}$, JIANMING ZHANG $^{3}$, MENGSHAN CHEN $^{4}$, \\ HONGJIE LI $^{1}$, SHANCHUAN ZHENG ${ }^{5}$ and JINGJING QUAN ${ }^{6}$ \\ ${ }^{1}$ Department of Oral Pathology, School and Hospital of Stomatology, Tianjin Medical University, Heping, \\ Tianjin 300070; ${ }^{2}$ Department of Oral Pathology, Peking University School and Hospital of Stomatology, \\ Haidian, Beijing 100081; ${ }^{3}$ Department of Stomatology, General Hospital of Tianjin Medical University, \\ Heping, Tianjin 300052; ${ }^{4}$ School of Public Health, Tianjin Medical University, Heping, Tianjin 300070; \\ ${ }^{5}$ Department of Stomatology, WuQing People's Hospital, Wuqing, Tianjin 301700; ${ }^{6}$ Guanghua School of Stomatology, \\ Hospital of Stomatology, Sun Yat-Sen University and Guangdong Provincial Key Laboratory of Stomatology, \\ Guangzhou, Guangdong 510080, P.R. China
}

Received March 15, 2017; Accepted January 23, 2018

DOI: $10.3892 / 01.2018 .8308$

\begin{abstract}
The seven-amino acid truncated (7ND) protein is an N-terminal deletion mutant of monocyte chemoattractant protein-1 (MCP-1) and it functions as a dominant-negative inhibitor. 7ND and wild-type MCP-1 form a heterodimer, which binds to MCP-1 receptors and inhibits monocyte chemotaxis. In the present study, the 7ND protein was cloned, expressed and purified. An MTT assay revealed that the proliferation of oral squamous cell carcinoma (OSCC) SCC25 cells was not affected following 3 days of treatment with synthetic 7 ND protein. Serial dilutions of the 7ND protein were tested for monocyte migration and osteoclast differentiation, and tartrate-resistant acid phosphatase staining demonstrated that significantly fewer osteoclasts were differentiated from cluster of differentiation $14^{+}\left(\mathrm{CD}^{+} 4^{+}\right)$monocytes using magnetic activated cell sorting. Immunofluorescence confirmed these results and significantly less F-actin staining was observed
\end{abstract}

Correspondence to: Dr Jianming Zhang, Department of Stomatology, General Hospital of Tianjin Medical University, 154 Anshan Road, Heping, Tianjin 300052, P.R. China

E-mail: zjming17@163.com

Dr Jingjing Quan, Guanghua School of Stomatology, Hospital of Stomatology, Sun Yat-Sen University and Guangdong Provincial Key Laboratory of Stomatology, 56 Lingyuan West Road, Guangzhou, Guangdong 510080, P.R. China

E-mail: quanjj3@mail.sysu.edu.cn

${ }^{*}$ Contributed equally

Key words: bone invasion, oral squamous cell carcinoma, monocyte chemoattractant protein-1,7ND, osteoclasts in 7ND-treated osteoclasts. Furthermore, bone invasion was examined by subcutaneously injecting SCC25 cells into the area overlaying the calvariae of nude mice. The results demonstrated that the average tumor volume of SCC25 cells with 7ND protein was similar to the average volume of tumors formed by untreated SCC25 cells. Flow cytometric analysis suggested that the $\mathrm{CD} 14^{+}$subpopulation in the bone marrow of 7ND-treated mice was reduced compared with that of untreated mice. Micro-computed tomography imaging revealed significantly less bone resorption in the calvariae injected with SCC25 cells plus the 7ND protein. Taken together, the results of the present study demonstrated the potential therapeutic value of the 7ND protein. The 7ND MCP-1 variant not only functions in vitro to inhibit osteoclast differentiation, but also reduces the progression of bone invasion by OSCC cells in vivo.

\section{Introduction}

Monocyte chemoattractant protein-1 (MCP-1), also known as chemokine ligand 2, is one of the chemokine family members (1). MCP-1 mediates neoplasm-induced osteoclastogenesis in different types of cancer, including myeloma, breast cancer and prostate cancer, due to its specific function, which is associated with osteoclast development and maturation (2). Kim et al (3) reported that MCP-1 was induced by receptor activator of nuclear factorkB ligand (RANKL), where it promoted human osteoclast differentiation. Furthermore, MCP-1-treated human peripheral blood mononuclear cells (PBMCs) formed tartrate-resistant acid phosphatase (TRAP)-positive multinucleated cells, suggesting that $\mathrm{MCP}-1$ promotes osteoclast fusion $(4,5)$.

In contrast to distant bone metastasis, local bone invasion is a common complication of oral squamous cell carcinoma (OSCC). Lesions of the gingiva, hard palate and retromolar 
trigone may involve the maxillary and/or mandibular bone (6). It is now clear that osteoclasts are involved in this resorption process (7). These osteoclasts differentiate at the bone surface and dissolve mineral components. Since osteoclasts serve important roles in the progression of bone invasion in OSCC, it is essential to identify an efficient therapeutic osteoclast target, which may improve clinical approaches.

Our previous study examined whether a decrease in MCP-1 expression would inhibit OSCC bone invasion (8). To begin with, MCP-1 protein and mRNA expression in OSCC tissues and several OSCC cell lines were confirmed. Subsequently, SCC25 cells were transfected with a dominant-negative variant of MCP-1 with 7-amino acid truncated (7ND) in the pcDNA vector (SCC25-7ND). Following this, it was revealed that $10 \%$ SCC25-7ND cell conditioned medium efficiently inhibited human osteoclast formation. By establishing an animal model of OSCC bone invasion, histological analysis identified significantly fewer SCC25-7ND osteoclasts within the calvariae compared with SCC25 cells. These results demonstrated the relevance of MCP-1 in the study of OSCC bone invasion and indicated that 7ND may be used as a blocking agent to inhibit MCP-1. Therefore, the present study aimed to utilize the synthetic 7ND protein in order to determine whether it inhibits in vitro differentiation of osteoclasts and whether it reduces SCC25 bone invasion in vivo.

\section{Materials and methods}

Reagents. Dulbecco's modified Eagle's medium (DMEM), $\alpha$-Modified Eagle Medium ( $\alpha$-MEM), fetal bovine serum (FBS), trypsin-EDTA, antibiotics (100 U/ml penicillin $\mathrm{G}$ and $100 \mathrm{mg} / \mathrm{ml}$ streptomycin) and phosphate buffered saline (PBS) were purchased from Thermo Fisher Scientific, Inc. (Waltham, MA, USA). The primary monoclonal mouse anti-human MCP-1 antibody was obtained from Abcam (cat. no. ab9858; Cambridge, MA, USA) and the primary fluorescein isothiocyanate (FITC) rat anti-mouse cluster of differentiation 14 (CD14) antibody was purchased from BD Biosciences (cat. no. 561710; San Jose, CA, USA). The horseradish peroxidase (HRP)-conjugated goat anti-mouse IgG secondary antibody was supplied by Bio-Rad Laboratories, Inc. (cat. no. STAR137P; Hercules, CA, USA). Recombinant human cytokines of colony stimulating factor1 (rhCSF1), RANKL (rhRANKL) and MCP-1 (rhMCP-1) were purchased from PeproTech, Inc. (Rocky Hill, NJ, USA), A TRAP staining kit was obtained from Sigma-Aldrich; Merck KGaA (Darmstadt, Germany).

Immunohistochemistry. OSCC tissue samples from 10 patients with bone invasion were examined in order to determine MCP-1 expression. Adjacent non-cancerous tissues from the same patients served as control. Following being fixed in $4 \%$ paraformaldehyde (Sigma-Aldrich; Merck KGaA) for 1 week at room temperature, serial paraffin-embedded tissue sections (5 $\mu \mathrm{m}$ thick) were dewaxed using xylene, rehydrated in a descending alcohol series (100, 85 and 75\% ethanol) and treated with $0.3 \%$ hydrogen peroxide in PBS. Antigen retrieval was performed by heating $\left(50^{\circ} \mathrm{C}\right)$ sections in a microwave oven (twice, for 4 min each time) with $0.2 \%$ citrate buffer $(\mathrm{pH}=6)$. Non-specific binding was blocked with $5 \%$ bovine serum albumin in PBS for 30 min at room temperature, and sections were subsequently incubated with the primary MCP-1 antibody (dilution, 1:100) overnight at $4^{\circ} \mathrm{C}$. Sections were then treated with Dako REAL ${ }^{\mathrm{TM}}$ EnVision $^{\mathrm{TM}} / \mathrm{HRP}$, Rabbit/Mouse reagent of the kit(horseradish peroxidase-conjugated polymer; cat. no. K5007; Envision Detection System; Dako; Agilent Technologies, Inc., Santa Clara, CA, USA) for $30 \mathrm{~min}$ at room temperature, followed by 3,3'-diaminobenzidine (DAB) detection solution for $1 \mathrm{~min}$ at room temperature. The primary antibody was replaced with non-immune serum (Wuhan Boster Biological Technologies, Ltd., Wuhan, China) as a negative control. All sections were counterstained with Mayer's hematoxylin for $3 \mathrm{~min}$ at room temperature, dehydrated and mounted with mounting medium. The final results were visualized with a light microscope (magnification, x200) and images were captured using a digital camera.

Cloning, protein expression and purification. The full-length 7ND gene was amplified using primers designed based on the full-length MCP-1 gene (GenBank: S71513.1, forward, 5'-TCGCGAGCTATAGAAGAATCA-3' and reverse, 5'-TGT TCAAGTCTTCGGAGTTTG-3'). The 7ND coding region was cloned into the pMCSG7 vector (9). The recombinant plasmid was sequenced and the plasmid harboring the 7ND gene was transformed into BL21 (DE3) E. coli. The cells were cultured in Luria-Bertani medium (Wuhan Boster Biological Technologies, Ltd.) containing ampicillin $(100 \mu \mathrm{g} / \mathrm{ml})$ at $37^{\circ} \mathrm{C}$ until the $\mathrm{OD}_{600}$ reached 0.8 . The culture was induced with $0.2 \mathrm{mmol} / 1$ iso-propyl- $\beta$-D-thiogalactoside for $20 \mathrm{~h}$ at $16^{\circ} \mathrm{C}$. Cells were harvested by centrifugation at $4^{\circ} \mathrm{C}(5,000 \mathrm{x}$ g for $5 \mathrm{~min}$ ), lysed by sonication and clarified by centrifugation at $4^{\circ} \mathrm{C}(12,000 \mathrm{x} \mathrm{g}$ for $20 \mathrm{~min})$. The supernatant was then applied to a nickel-nitrilo-triacetic acid (Ni-NTA) resin gravity column (Qiagen, Inc., Valencia, CA, USA) that had been previously equilibrated with PBS (137 mmol/l NaCl, $2.7 \mathrm{mmol} / \mathrm{l} \mathrm{KCl}$, $50 \mathrm{mmol} / 1 \mathrm{Na}_{2} \mathrm{HPO}_{4}$ and $10 \mathrm{mmol} / 1 \mathrm{KH}_{2} \mathrm{PO}_{4} ; \mathrm{pH}$ 7.4). The column was washed with $100 \mathrm{ml}$ PBS, followed by washing with $100 \mathrm{ml} 20 \mathrm{mmol} /$ limidazole in PBS and elution with $300 \mathrm{mmol} / \mathrm{l}$ imidazole in PBS. Following buffer exchange, the His-tag was cleaved by tobacco etch virus treatment. Uncut protein was separated by a second Ni-affinity chromatography. Protein-containing fractions were pooled, concentrated and loaded onto a Superdex G200 size exclusion chromatography column (GE Healthcare, Chicago, IL, USA), equilibrated with $20 \mathrm{mmol} / \mathrm{l}$ Tris- $\mathrm{HCl}$ at $\mathrm{pH} 8.0,150 \mathrm{mmol} / \mathrm{l} \mathrm{NaCl}$ and $2 \mathrm{mmol} / \mathrm{l}$ DTT.

Western blot analysis. Total protein was extracted using radioimmunoprecipitation lysis buffer (Thermo Fisher Scientific, Inc.) and clarified by centrifugation at $4^{\circ} \mathrm{C}$ $(12,000 \mathrm{x} g$ for $20 \mathrm{~min})$. Protein concentration was determined using a bicinchoninic acid protein assay kit (Pierce; Thermo Fisher Scientific, Inc.). A total of $40 \mu \mathrm{g}$ protein was separated by $10 \%$ SDS-PAGE (Bio-Rad Laboratories, Inc.), prior to being transferred onto polyvinylidene difluoride membranes (EMD Millipore, Billerica, MA, USA) and subsequently blocked with $5 \%$ dry skimmed milk in Tris-buffered saline for $1 \mathrm{~h}$ at room temperature. The membranes were incubated with an HRP-conjugated human anti-His antibody (cat. no. ab219465; 1:10,000; Abcam) 
overnight at $4^{\circ} \mathrm{C}$, washed twice with PBS and subsequently incubated with HRP-conjugated goat anti-mouse IgG secondary antibodies for $1 \mathrm{~h}$ (cat. no. STAR137P; 1:3,000; Hercules) at room temperature. Protein bands were subsequently detected and visualized using a Super Signal WestPico chemiluminescent substrate (Thermo Fisher Scientific, Inc.).

Cell lines and culture. The OSCC SCC 25 cell line was obtained from American Type Culture Collection (Manassas, VA, USA). SCC25 cells were cultured in DMEM, supplemented with $10 \%$ FBS and antibiotics (100 U/ml penicillin $\mathrm{G}$ and $100 \mathrm{mg} / \mathrm{ml}$ streptomycin) at $37^{\circ} \mathrm{C}$ in an incubator containing $5 \% \mathrm{CO}_{2}$ and $20 \% \mathrm{O}_{2}$.

Cell proliferation assay. SCC 25 cells were seeded onto 96-well plates $\left(5 \times 10^{3}\right.$ cells/well) and were allowed to attach overnight, prior to being treated with $7 \mathrm{ND}$ protein $(0,25$, 50,100 or $200 \mathrm{ng} / \mathrm{ml}$ ) for 1-3 days. A volume of $20 \mu \mathrm{l}$ MTT ( $5 \mathrm{mg} / \mathrm{ml}$; Thermo Fisher Scientific, Inc.) was added to each well for $4 \mathrm{~h}$ at $37^{\circ} \mathrm{C}$. Following removal of solution and addition of dimethyl sulfoxide (150 $\mu \mathrm{l}$ /well; Sigma-Aldrich; Merck $\mathrm{KGaA}$ ) to dissolve the purple-formazan the absorbance was read at $590 \mathrm{~nm}$ on a BioTek plate reader (Beckman Coulter, Inc., Brea, CA, USA).

Magnetic activated cell sorting (MACS) of $\mathrm{CDI}^{+}$monocytes. Human PBMCs were isolated from the blood of healthy volunteers using BD vacutainer cell preparation tubes containing sodium citrate, as previously described (8). Following centrifugation at $1,500 \mathrm{x} \mathrm{g}$ for $30 \mathrm{~min}$ of room temperature, the cell layer on top of the Ficoll-Paque was collected, resuspended in $10 \mathrm{ml} \alpha$-MEM and centrifuged ( $225 \mathrm{x} \mathrm{g}$ for $10 \mathrm{~min}$ ) at room temperature. $\mathrm{CD} 14^{+}$monocytes were purified by incubation with MACS CD14 ${ }^{+}$microbeads (Miltenyi Biotec, Inc., Cambridge, MA, USA) for $15 \mathrm{~min}$ at $4^{\circ} \mathrm{C}$. Cells were subsequently washed in $\mathrm{CD} 14^{+}$isolation buffer $(0.5 \%$ fetal calf serum and $2 \mathrm{mM}$ EDTA; $\mathrm{pH}=8$ ) and passed through a MACS magnetic cell separator (Miltenyi Biotec, Inc.). $\mathrm{CD} 14^{+}$monocytes were collected and utilized for subsequent experiments.

Migration assay. Transwell inserts (5 $\mu \mathrm{m}$ pore; Corning Incorporated, Corning, NY, USA) were used as previously described (10). Prior to loading, CD14 ${ }^{+}$monocytes $\left(1 \times 10^{5}\right.$ cells $\left./ \mathrm{ml}\right)$ were incubated for $1 \mathrm{~h}$ with various concentrations of $7 \mathrm{ND}$ protein $(0,25,50$ or $100 \mathrm{ng} / \mathrm{ml})$, and were subsequently seeded into the upper chamber [serum-free culture medium of $\alpha$-modified-minimum essential medium $(\alpha-M E M)$; Thermo Fisher Scientific, Inc.] of Transwell inserts, while $600 \mu 1$ complete medium of $\alpha$-MEM with $10 \%$ FBS (Thermo Fisher Scientific, Inc.) containing $10 \mathrm{ng} / \mathrm{ml}$ rhMCP-1 was placed into the lower chamber. Following $3 \mathrm{~h}$ of incubation, non-migrating cells were scraped from the upper chamber, and migrated cells were stained with Hoechst 33342 (Sigma-Aldrich; Merck KGaA) at room temperature for $5 \mathrm{~min}$ and were observed using a fluorescence microscope (magnification, x200). Four fields were randomly selected and non-overlapping images were captured for each of three triplicate culture wells. In each image, the total number of stained cells was counted by two independent assessors.

Osteoclast differentiation assay. $\mathrm{CD}^{+} 4^{+}$monocytes were seeded onto 24 -well plates $\left(1 \times 10^{5}\right.$ cells/well) with $600 \mu 1$ medium ( $\alpha$-MEM; pH 7.4; containing 10\% FBS and $1 \%$ penicillin/streptomycin), supplemented with rhCSF1 $(25 \mathrm{ng} / \mathrm{ml})$ and rhRANKL $(40 \mathrm{ng} / \mathrm{ml})$ to induce osteoclast differentiation. Groups were arranged as follows: Group 1, $\mathrm{CD}_{1} 4^{+}$monocytes with $\mathrm{rhCSF} 1(25 \mathrm{ng} / \mathrm{ml})$ and rhRANKL (40 ng/ml); and Group 2, CD14 ${ }^{+}$monocytes with rhCSF1 $(25 \mathrm{ng} / \mathrm{ml})$, rhRANKL (40 ng/ml) and 7ND protein $(50 \mathrm{ng} / \mathrm{ml})$. Medium was changed every 3 days and mature osteoclasts appeared in one week. Osteoclasts were subsequently fixed in $10 \%$ formalin for $5 \mathrm{~min}$ at room temperature. Staining of TRAP was used to characterize osteoclasts $(5 \mathrm{~min}$ at room temperature). TRAP-positive cells with three or more nuclei were considered to be multinucleated osteoclasts. Rhodamine-conjugated phalloidin (Thermo Fisher Scientific, Inc.) was used to label F-actin and DAPI staining (Thermo Fisher Scientific, Inc.) was used to visualize nuclei for $5 \mathrm{~min}$ at room temperature by using a fluorescence microscope (magnification, x200). Four fields were randomly selected and non-overlapping images were captured for each of three triplicate culture wells. In each image, the total number of TRAP-positive multinucleated osteoclasts and the number of their F-actin rings were counted by two independent assessors.

In vivo animal model of OSCC bone invasion. A total of $18 \mathrm{BALB} /-\mathrm{c}$ nude mice (female, 6-7 weeks, 15-18 g) were obtained from the animal resources center (Sun Yat-sen University, Guangzhou, China), housed in the animal facility and cared for by animal housing staff. The housing conditions included specific pathogen free animal rooms $\left(20-26^{\circ} \mathrm{C}\right.$, 20-50 Pa, 12/12 h dark-light cycle, positive atmosphere/clean area and negative atmosphere/affected area). The holding food and water were checked and prepared by animal housing staff each day. All protocols were reviewed and approved by the University Ethics Committee of Sun Yat-Sen University (2016-334QX). The humane endpoints were conditions which severely affect the normal diet or breath of the nude mice, absolute values included the fast growth of tumors i.e., (if tumor volume $\geq 3.26 \mathrm{~cm}^{3}$, or weight loss i.e., the body weight is $\leq 10 \mathrm{~g})$. OSCC SCC25 cells $\left(5 \times 10^{6} / 100 \mu \mathrm{l}\right)$ were subcutaneously injected into the area overlaying the calvaria when the mice were 6-7 weeks old. Mice were randomly divided into three groups ( $\mathrm{n}=6 /$ group): The negative control group (Group 1) received PBS; the positive control group (Group 2) received SCC25 cells; and the experimental group (Group 3) received SCC25 cells plus 7ND protein $(30 \mu \mathrm{g} / \mathrm{ml})$. $7 \mathrm{ND}$ protein was subcutaneously injected into the same location overlaying the calvaria every other day. All animals were sacrificed after 4 weeks, and tumors and calvariae were fixed in 4\% paraformaldehyde (Sigma-Aldrich; Merck KGaA) for 1 week at room temperature. The maximum tumor volume observed in any of the mice was $3.26 \mathrm{~cm}^{3}$.

Micro-computed tomography $(\mu C T)$ imaging. All calvariae were surgically removed from PBS-treated control, SCC25 and SCC25+7ND tumor-bearing nude mice, were fixed 
in $70 \%$ ethanol for 1 day at room temperature and scanned using a $\mu \mathrm{CT}$ instrument (SCANCO Medical AG, Brüttisellen, Switzerland). $\mu \mathrm{CT}$-analyzer software (version 3.0; Volume Graphics GmbH, Heidelberg, Germany) was used to analyze the calvarial structure using the global segmentation method (8). Two-dimensional images were used to generate three-dimensional reconstruction. The calvarial area was outlined for analysis and quantification as previously described (8). The amount of resorbed bone was defined as the percentage of resorbed bone volume divided by the total bone volume.

Flow cytometry. Bone marrow cells (BMCs) of each mouse were extracted from the tibia on week 4 , and blocked in $5 \%$ bovin serum albumin (Wuhan Boster Biological Technologies, Ltd.) for $15 \mathrm{~min}$ at room temperature. The $\mathrm{CD} 14^{+}$subpopulation of BMCs was evaluated by incubating $1 \times 10^{6}$ cells with FITC rat anti-mouse CD14 antibody (cat. no. 561710; BD Biosciences, San Jose, CA, USA) in PBS (dilution, 1:100) at $4^{\circ} \mathrm{C}$ for $30 \mathrm{~min}$. To wash off excess antibody following staining, PBS was added and subsequently centrifuged cell pellet was obtained (225 x g, 5 min, room temperature). Flow cytometry analysis (FACS) was performed on a FACScan flow cytometer (BD Biosciences) as previously reported (8). The unstained cells were gated out and data acquisition with analysis was performed using FlowJo LLC software (version 7.6; FlowJo LLC, Ashland, OR, USA).

Histological and immunohistochemical analysis. Subsequent to being fixed in $4 \%$ paraformaldehyde (Sigma-Aldrich; Merck $\mathrm{KGaA}$ ) for 1 week at room temperature, tumor specimens were embedded in paraffin using a tissue processor. Serial 5- $\mu \mathrm{m}$ paraffin-embedded sections were cut on a rotary microtome (Leica Microsystems, Inc., Buffalo Grove, IL, USA) and were stained with $\mathrm{H} \& \mathrm{E}$ at room temperature for $1 \mathrm{~h}$. Immunohistochemical staining of sections was performed by incubating serial sections with the MCP-1 primary antibody (dilution, $1: 100$ ) overnight at $4^{\circ} \mathrm{C}$, followed by incubation $(1 \mathrm{~h})$ with Dako REAL ${ }^{\mathrm{TM}}$ EnVision $^{\mathrm{TM}} / \mathrm{HRP}$, Rabbit/Mouse reagent (horseradish peroxidase-conjugated polymer; cat. no. K5007; Envision Detection System; Dako; Agilent Technologies, Inc.) at room temperature and DAB (Dako; Agilent Technologies, Inc.) staining for $3 \mathrm{~min}$ at room temperature. Specimens treated with non-immune serum served as a negative control.

All tumor-bearing calvaria were decalcified in 10\% EDTA $(\mathrm{pH}=7.4)$ for 2 weeks at room temperature, prior to being processed for paraffin embedding. Serial $5 \mu \mathrm{m}$ sections were stained with H\&E and TRAP separately, each for $1 \mathrm{~h}$ at room temperature. Analysis of the number of TRAP-positive osteoclasts at the tumor-bone interface was performed as previously described (8). For each section, an area of $2 \mathrm{~mm}^{2}$ with the tumor-bone interface was defined for counting the number of osteoclasts. Four fields of this area were randomly selected and the number of TRAP-positive osteoclasts was counted using a light microscope (magnification, x200).

Statistical analysis. Results are presented as the mean \pm standard error of the mean of $\geq 3$ independent
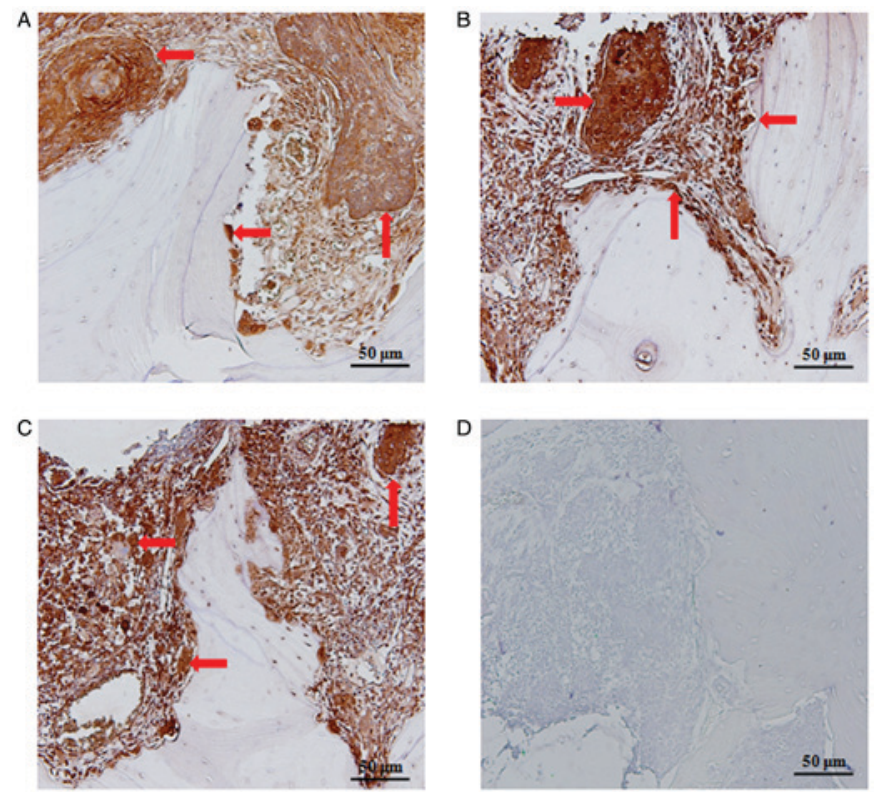

Figure 1. MCP-1 staining in OSCC tissues with bone invasion. (A-C) MCP-1 protein localized to the cytoplasm of osteoclasts and OSCC cells at the tumor-bone interface. (D) Control sections exhibiting negative staining. Arrow, 3,3'-diaminobenzidine; scale bar $=50 \mu \mathrm{m}$. MCP-1, monocyte chemoattractant protein-1; OSCC, oral squamous cell carcinoma.

experiments. Data analysis was performed using SPSS software (version 20.0; IBM Corp., Armonk, NY, USA). Student's t-test was used to compare two means. One-way analysis of variance, followed by Student-Newman-Keuls test, was applied to compare two or more means. $\mathrm{P}<0.05$ was considered to indicate a statistically significant difference.

\section{Results}

MCP-1 protein is expressed in OSCC tissues with bone invasion. Strong staining of the MCP-1 protein was observed in 10 of the OSCC tissue samples. Immunohistochemistry (IHC) demonstrated that MCP-1 was localized to osteoclasts and the cytoplasm of OSCC cells at the tumor-bone interface (Fig. 1A-C). Control sections did not exhibit any staining (Fig. 1D).

In vitro synthesis and validation of the 7ND protein. Based on the standard protocol (9), the 7ND protein was cloned, expressed and purified in vitro. Uncut protein was separated by a second Ni-affinity chromatography column and SDS-PAGE prior to and following purification (Fig. 2A-B). $7 \mathrm{ND}$ is an N-terminal deletion mutant of MCP-1 and lacks amino acids 2 to 8 , and a schematic structural diagram of MCP-1 and 7ND is presented in Fig. 2C. Western blot analysis also confirmed the size of the synthetic 7ND protein (Fig. 2D).

The 7ND protein does not affect SCC25 cell proliferation. Results of the MTT assay indicated that the 7ND protein did not affect SCC25 cell proliferation following 3 days of treatment at various concentrations (Fig. 3A-C). No significant differences were observed between pre- and post-treatment measurements at any concentration. 


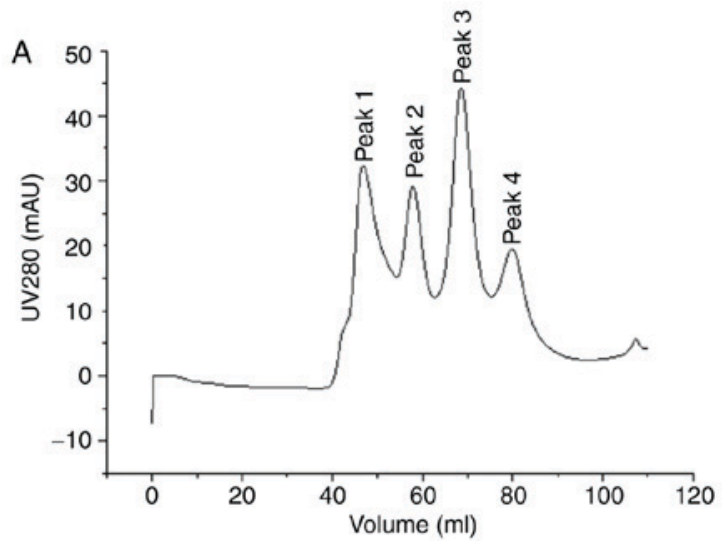

B

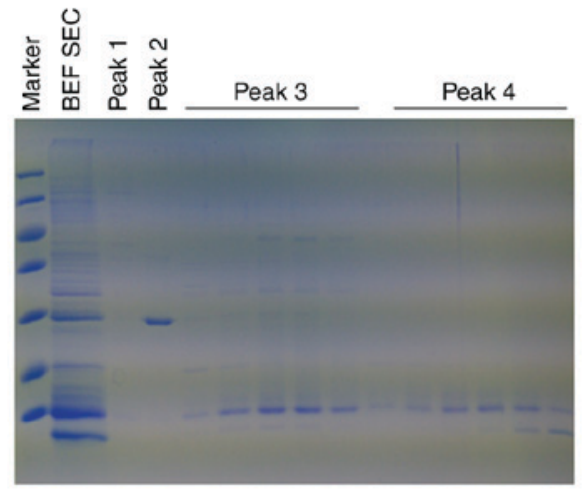

C

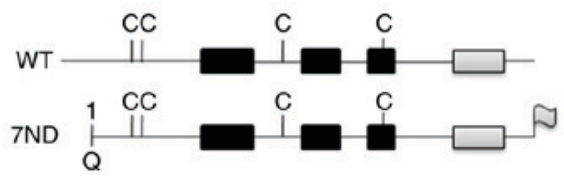

D

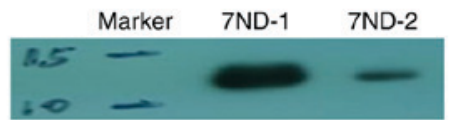

Figure 2. Expression, purification and validation of synthetic 7ND protein (A) Separation of uncut protein by a second Ni-affinity chromatography; (B) SDS-PAGE confirmation of different protein regions prior to and following purification. (C) A schematic diagram of monocyte chemoattractant protein-1 and 7ND; (D) western blot analysis confirming the size of the $7 \mathrm{ND}$ protein. WT, wild-type; BEF-SEC, before separation, uncut protein.

$7 N D$ inhibits the migration of $\mathrm{CD}^{+} 4^{+}$monocytes. A migration assay was performed in order to determine the effects of the $7 \mathrm{ND}$ protein on the migration of $\mathrm{CD} 14^{+}$monocytes. Compared with control $\mathrm{CD} 14^{+}$monocytes, various concentrations of the $7 \mathrm{ND}$ protein $(25,50$ and $100 \mathrm{ng} / \mathrm{ml})$ efficiently inhibited $\mathrm{CD} 14^{+}$monocyte migration (Fig. 4A-B).

The $7 \mathrm{ND}$ protein inhibits the differentiation of $\mathrm{CD}^{+} 4^{+}$monocytes into osteoclasts. The differentiation of osteoclasts from the positive control group with $\mathrm{CD} 14^{+}$monocytes was induced by the addition of rhCSF1 and rhRANKL for 3-4 days in culture (Fig. 5A-B). The effect of the 7ND protein $(50 \mathrm{ng} / \mathrm{ml})$ on osteoclast differentiation was examined, and TRAP staining revealed that there were significantly fewer osteoclasts in the presence of $7 \mathrm{ND}$ (Fig. 5A-B). Immunofluorescence also revealed significantly less staining of F-actin in the presence of 7ND (Fig. 6A-B).

The 7ND protein reduces the SCC25 cell-induced resorption area of calvariae. An animal model of SCC25 cell
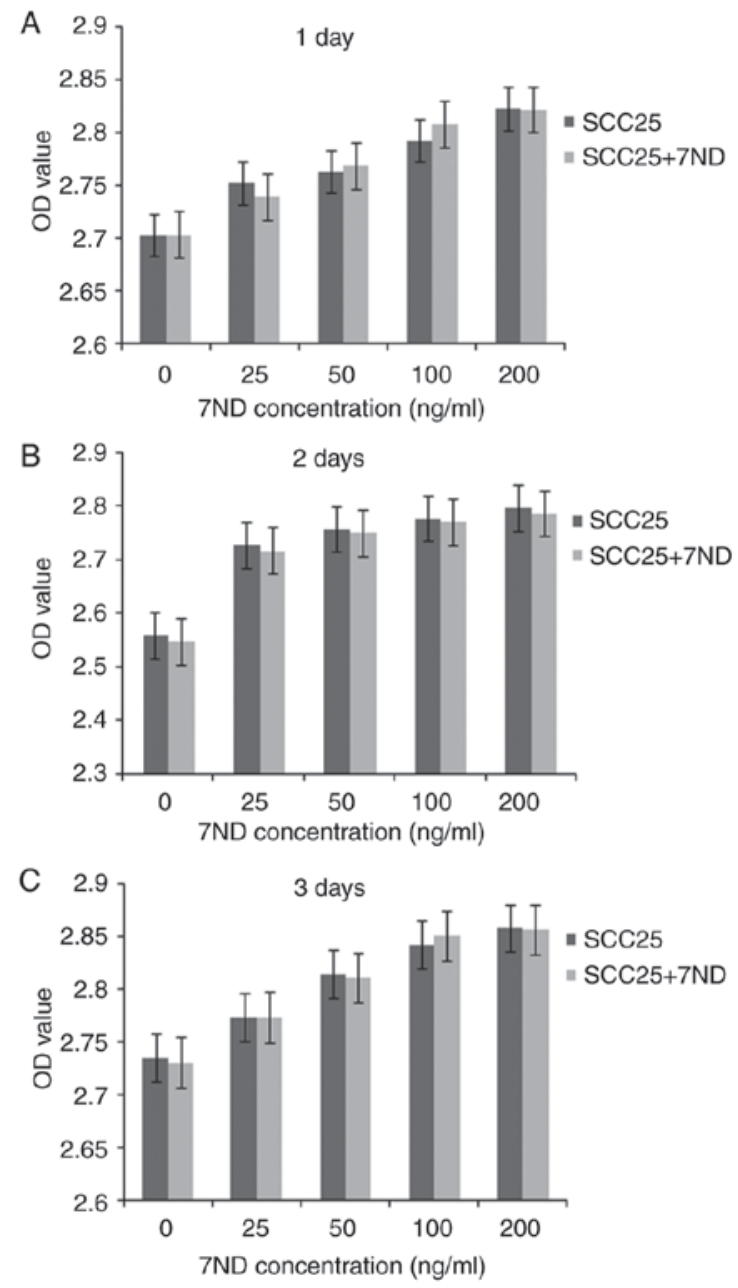

Figure 3. 7ND protein did not affect SCC25 cell proliferation. MTT results demonstrated that SCC25 cell proliferation was not affected after (A) 1, (B) 2 or (C) 3 days of 7ND treatment $(25,50,100$ or $200 \mathrm{ng} / \mathrm{ml})$. Data are presented as the mean \pm the standard error of the mean of three independent experiments. OD, optical density.

bone invasion was utilized as previously described (8), and tumor cells were injected subcutaneously through the center of the calvaria. Data regarding the tumor volume (width $\mathrm{x}$ length $\mathrm{x}$ depth) were recorded at week 4 , and the results demonstrated that the average tumor volume was similar in the SCC25+7ND group and the SCC25 group (Fig. 7A-B). $\mu \mathrm{CT}$ analysis revealed a significant decrease in the bone resorption area of the calvaria in the $\mathrm{SCC} 25+7 \mathrm{ND}$ group (Fig. 7A-B).

The CD14+ subpopulation of BMCs is reduced in mice injected with $7 N D$. FACS analysis revealed that the CD14+ ${ }^{+}$subpopulation of BMCs was reduced in mice of the SCC25+7ND group (11.5\%; Fig. 8C), compared with the group of mice that received SCC25 cells only (13.6\%, Fig. 8B). A significant difference was observed between these two groups.

MCP-1 protein is localized to the cytoplasm of tumor cells in vivo. Well-differentiated OSCC formed in the two groups following injection with SCC25 cells with or without 7ND protein (Fig. 9). Immunohistochemistry was performed in order to examine MCP-1 protein localization, and expression 
A
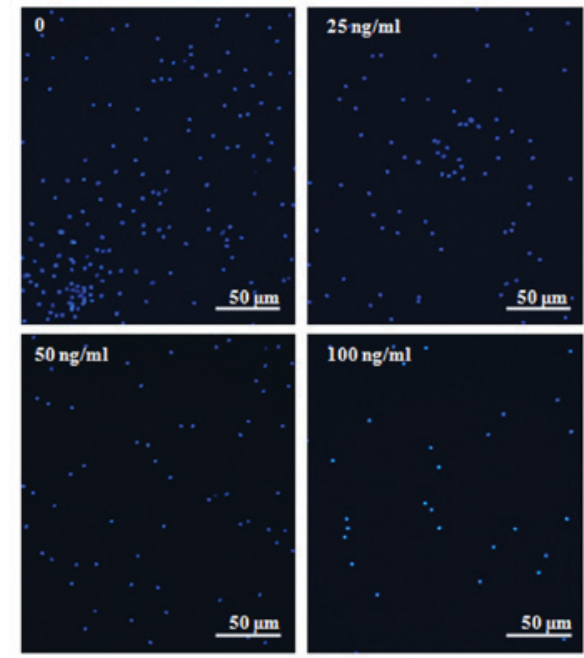

B

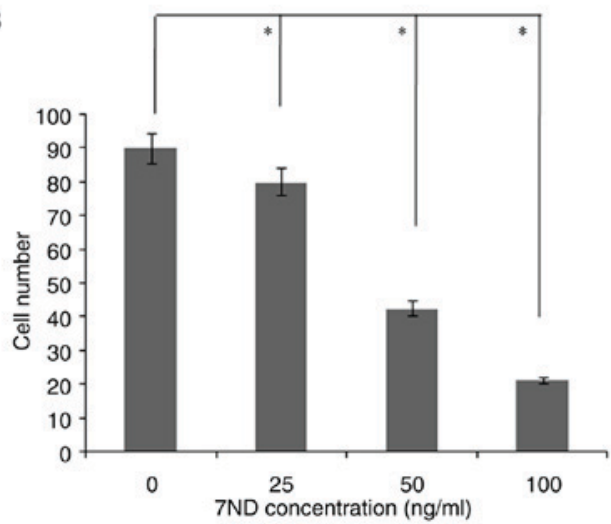

Figure 4. 7ND protein inhibited the migration of $\mathrm{CD} 14^{+}$monocytes. (A) Immunofluorescence demonstrated that various concentrations of $7 \mathrm{ND}$ protein $(25,50100 \mathrm{ng} / \mathrm{ml})$ efficiently inhibited the migration of CD14 ${ }^{+}$monocytes. (B) Quantification of the number of monocytes in 4 randomly selected fields. Data are presented as the mean \pm the standard error of the mean of three independent experiments. ${ }^{*} \mathrm{P}<0.05 . \mathrm{CD}$, cluster of differentiation.

was revealed to be primarily in the cytoplasm and the cell membrane of tumor cells (Fig. 9).

$7 N D$ decreases the number of osteoclasts in bone resorption lacunae. The two groups revealed osteoclast accumulation in the resorption lacunae (Fig. 10A). TRAP staining was used to locate and count osteoclasts. Results revealed significantly fewer osteoclasts localized to the tumor-bone interface in the SCC25+7ND group, compared with the SCC25 group (Fig. 10A-B).

\section{Discussion}

7ND is an N-terminal deletion mutant of MCP-1 (11). 7ND and wild-type MCP-1 form a heterodimer and bind to the MCP-1 receptor to inhibit monocyte chemotaxis (12). Ikeda et al (13) first suggested using the 7ND protein for skeletal muscle-directed in vivo electroporation and, in 2001, reported that transgenic mice expressing 7ND blocked the $\mathrm{C}-\mathrm{C}$ motif chemokine receptor 2 pathway in vivo (14). Blockage of endogenous MCP-1 activity has also been demonstrated to inhibit tumor-associated vessel formation and early proliferation of melanoma cells in nude mice (15).
A

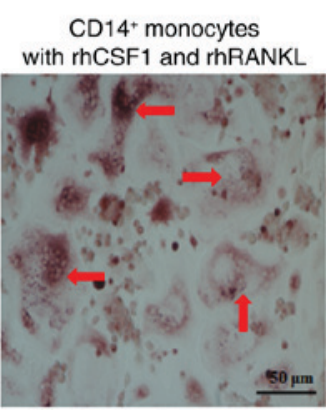

CD14+ monocytes with rhCSF1 and rhRANKL plus $7 \mathrm{ND}$ protein $(50 \mathrm{ng} / \mathrm{ml})$

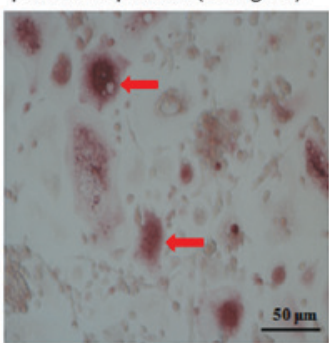

B

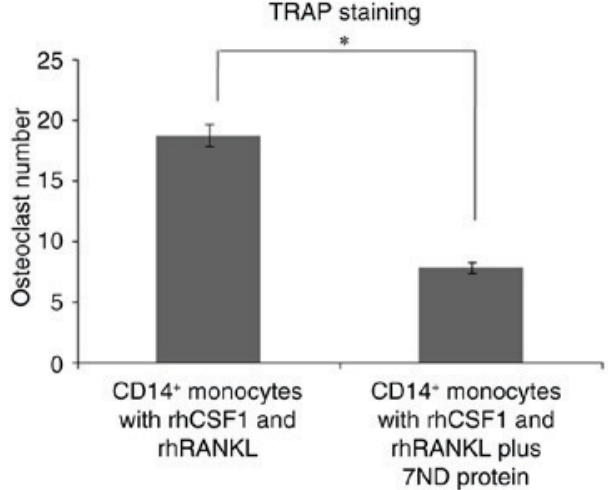

Figure 5. 7ND protein inhibited osteoclast differentiation from CD14 monocytes. (A) TRAP staining demonstrated that 7ND protein treatment $(50 \mathrm{ng} / \mathrm{ml})$ resulted in less osteoclast differentiation. (B) Quantification of osteoclast numbers was performed in 4 randomly selected fields. Data are presented as the mean \pm the standard error of the mean of three independent experiments. ${ }^{*} \mathrm{P}<0.05$. CD, cluster of differentiation; rhCSF1, recombinant human cytokines of colony stimulating factor 1 ; rhRANKL, recombinant human receptor activator of nuclear factor $\kappa \mathrm{B}$ ligand; TRAP, tartrate-resistant acid phosphatase.

Recent studies on the 7ND protein have revealed promising results, particularly for total joint replacement in end-stage arthritis (16-19). Yao et al (16) demonstrated that the 7ND protein decreased MCP-1-induced migration of THP-1 macrophage cells in a dose-dependent manner (16). A therapeutic strategy of local 7ND delivery at the implant site was further confirmed by the same group (17). By embedding the particles into the space between the periosteum and the calvaria bone, mice were treated with local injection of the 7ND protein. Compared with the PBS-treated control group, 7ND treatment significantly decreased particle-induced osteolysis, and led to an increase in bone volume and mineral density. The same group also reported the development of a biodegradable, layer-by-layer (LBL) coating platform, which permits efficient loading and controlled release of 7ND from the surface of orthopedic implants $(18,19)$. The LBL technique is suitable for an irregularly shaped material surface, and accomplishes higher drug loading and more controlled release. This is particularly useful for the rehabilitation of defective maxilla and/or mandible caused by OSCC. Compared with systemic treatment, the local delivery system has several advantages. The most important point is to retain the biological activity of synthesized protein, which may disturb the progression of local bone invasion.

In the present study, the 7ND protein was cloned, expressed and purified, based on the structure of MCP-1 and the 7ND protein. The results of SDS-PAGE and western blot analysis 
A

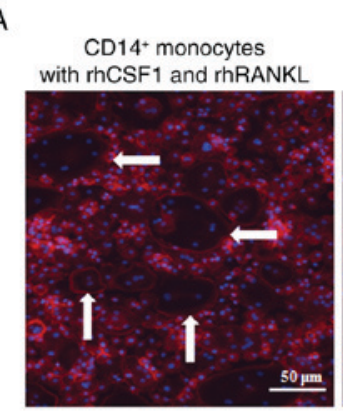

B

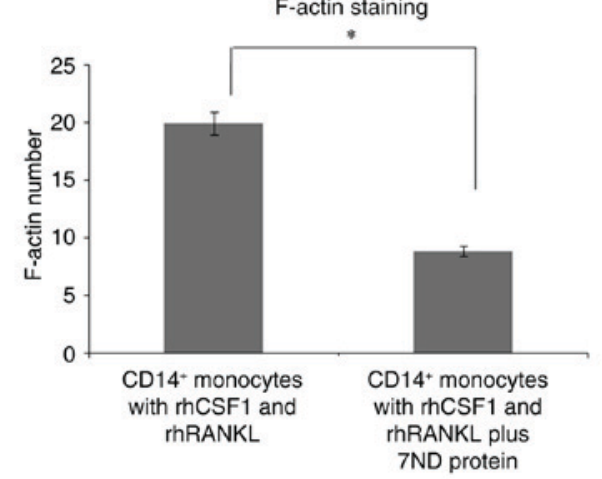

Figure 6. 7ND protein inhibited the formation of F-actin in osteoclasts. (A) Immunofluorescence revealed decreased staining of F-actin in cells treated with 7ND protein. (B) Quantification of the number of F-actin-stained cells was performed in 4 randomly selected fields. Data are presented as the mean \pm the standard error of the mean of three independent experiments. "P<0.05. CD, cluster of differentiation; rhCSF1, recombinant human cytokines of colony stimulating factor 1 ; rhRANKL, recombinant human receptor activator of nuclear factor $\mathrm{\kappa B}$ ligand.

confirmed the expression of the 7ND protein. Considering that it works as a dominant negative inhibitor of MCP-1, various concentrations of the 7ND protein were examined and the results revealed that 7ND efficiently inhibited the transmigration of $\mathrm{CD}_{1} 4^{+}$monocytes, with their differentiation into osteoclasts. These results confirmed the basic function of the synthetic $7 \mathrm{ND}$ protein in vitro, which is the basis for animal experiments involving 7ND injection in vivo. Furthermore, the synthetic 7ND protein did not have inhibitory effects on SCC25 tumor cell proliferation; therefore, it was reasonable to inject these tumor cells with the 7ND protein in vivo. Previous studies have attempted to modify the structure of the 7ND protein in order to improve its efficiency. Severin et al (20) constructed the 7ND-Fc fusion protein in order to extend its half-life. It was revealed that 7ND-Fc had antagonistic activity in experimental autoimmune encephalomyelitis.

Researchers have aimed to identify suitable biomarkers for OSCC bone invasion. Efficient markers to quickly identify the presence of bone invasion and effective targets to successfully treat patients are required. Russmueller et al (21) demonstrated that the upregulation of osteoprotegrin expression was associated with bone invasion, poor tumor regression and decreased long-term survival in 93 patients with OSCC. Tada et al (22) evaluated the effects of an NF- $\mathrm{KB}$ inhibitor (IMD-0560) using a mouse model of jaw bone invasion. It was demonstrated that IMD-0560 protected against zygoma and mandible destruction by SCCVII mouse OSCC cells. Furthermore, Hsu et al (23) reported the combined use of radiation and a multi-kinase
A
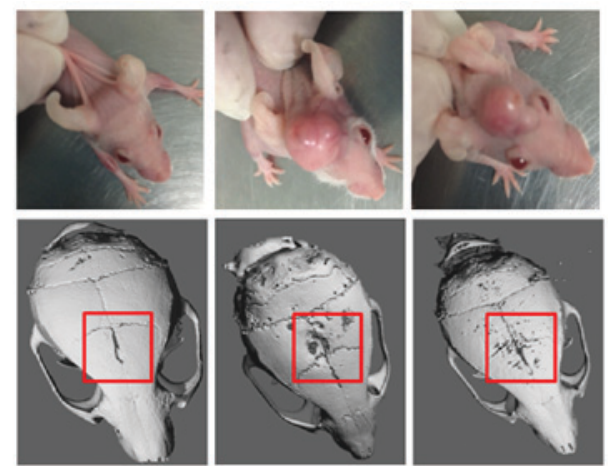

B

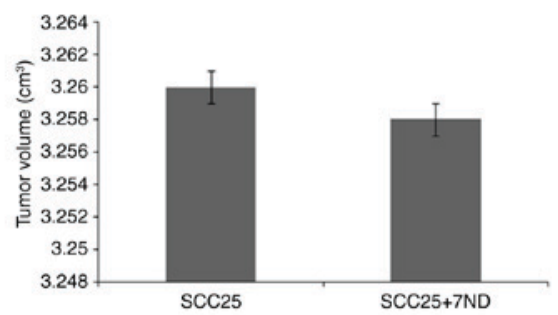

C

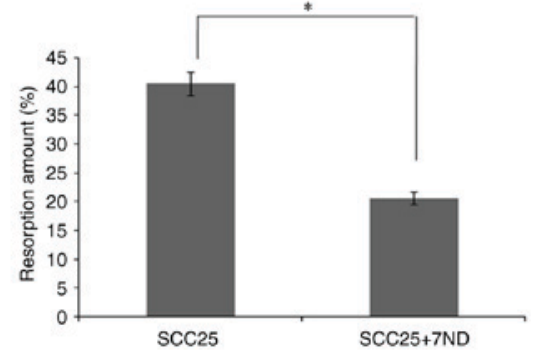

Figure 7. $\mu \mathrm{CT}$ analysis of bone resorption area within the calvaria. (A) Images of tumors were captured in week 4 and the same area of calvaria was outlined for $\mu \mathrm{CT}$ analysis. (B) The average tumor volume in the SCC25 cells plus $7 \mathrm{ND}$ injection group was similar to that in the SCC25 only group, while a (C) significantly smaller amount of resorbed bone was observed in the calvaria of the former group. Phosphate-buffered saline injection served as a negative control with an intact area of calvaria. Data are presented as the mean \pm the standard error of the mean of 6 mice for each group. ${ }^{*} \mathrm{P}<0.05$ $\mu \mathrm{CT}$, micro-computed tomography.

inhibitor of the extracellular signal-regulated kinase-NF- $\mathrm{BB}$ signaling pathway in an in situ human OSCC-bearing mouse model, and Sorafenib suppressed radiation-induced NF- $\mathrm{KB}$ activity and its downstream proteins.

Although the animal model used in the present study was distinct from those used in the afore mentioned studies, the advantages of calvariain jection animal models have been discussed previously (8). In the present study, the progression of OSCC bone invasion was reduced following 7ND treatment and significantly fewer osteoclasts accumulated at the tumor-bone interface, compared with the control group of untreated SCC25 cells. Therefore, the results of the present study suggested that 7ND has an inhibitory function in vivo, where it may circulate in the blood to inhibit monocyte migration in order to efficiently inhibit osteoclast differentiation. This observation was also confirmed by flow cytometric analysis, since the CD14+ subpopulation of BMCs was significantly reduced in the mice in the SCC25+7ND group, compared with the group of mice that received SCC25 cells only.

In conclusion, the results of the present study demonstrated the potential value of the 7ND protein in reducing the progression of 

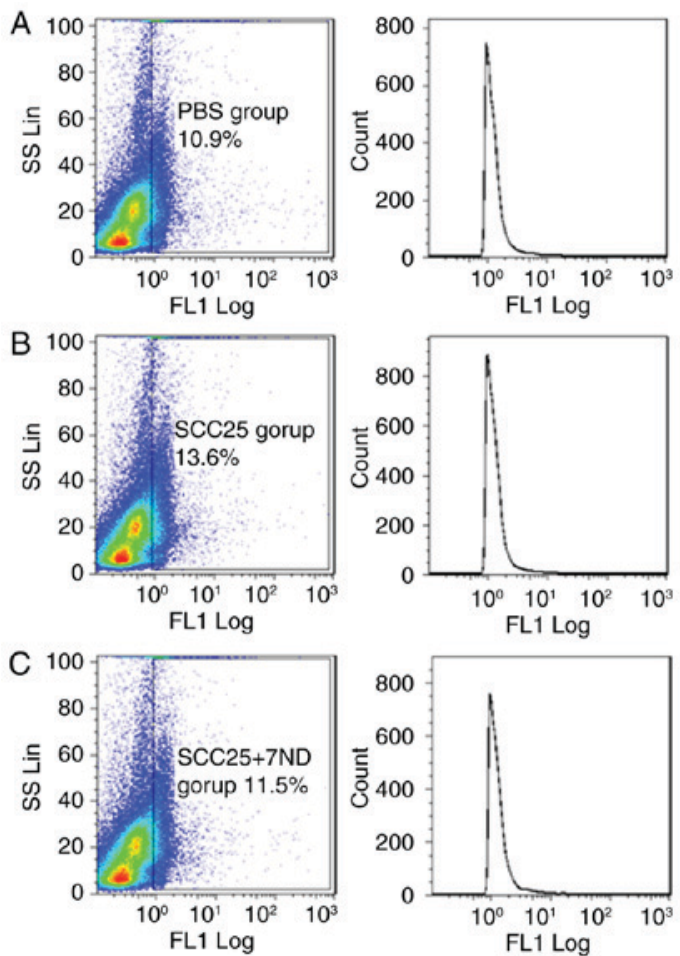

Figure 8. Fluorescence-activated cell sorting analysis of $\mathrm{CD}_{14}{ }^{+}$subpopulation. (A) The CD14+ subpopulation of BMCs in the phosphate-buffered saline control group was $10.9 \%$. (B) The group who received SCC25 cells only was $13.6 \%$. (C) The $\mathrm{CD} 14^{+}$subpopulation of BMCs was significantly reduced in mice of the SCC25+7ND group (11.5\%). Data are presented as the mean \pm the standard error of the mean of 6 mice for each group. $\mathrm{CD}$, cluster of differentiation; BMCs, bone marrow cells.

OSCC bone invasion. The MCP-1 variant, 7ND, not only works in vitro to inhibit osteoclast differentiation, but also reduces the progression of osteoclastic bone invasion in vivo.

\section{Acknowledgements}

The present study was supported by the Foundation of Sun Yat-Sen University (grant no. 13 ykpy 41), the Medical Scientific Research Foundation of Guangdong Province (grant no. B2014164) and the National Natural Science Foundation of China (grant no. 81500839).

\section{Funding}

No funding was received.

\section{Availability of data and materials}

The datasets generated and analyzed in the present study are included in this published article.

\section{Authors' contributions}

SL contributed to the work of immunohistochemistry and cell culture; $\mathrm{CZ}$ contributed to the work of $7 \mathrm{ND}$ cloning, expression, purification and validation; MC, HL and SZ contributed to the work of histological staining and data analysis; JZ contributed to the work of
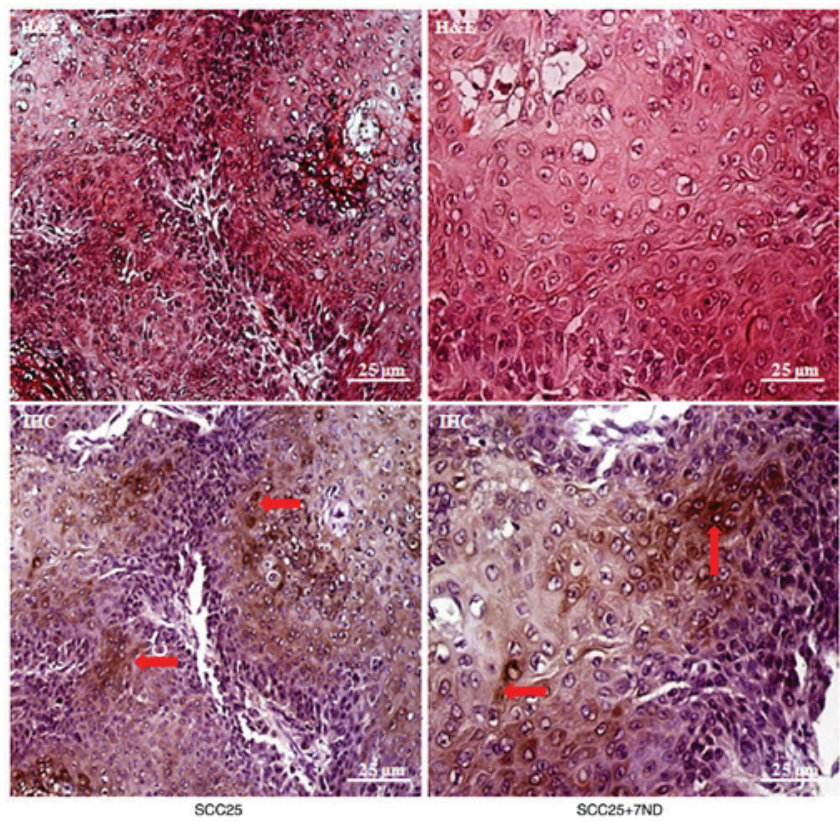

Figure 9. MCP-1 protein staining in tumor cells. H\&E staining revealed that well-differentiated OSCC had formed in the two groups. MCP-1 protein localized to the cytoplasm and cell membrane of the tumor cells. Arrow, 3,3'-diaminobenzidine; scale bar $=25 \mu \mathrm{m}$. MCP-1, monocyte chemoattractant protein-1; SCC, squamous cell carcinoma.
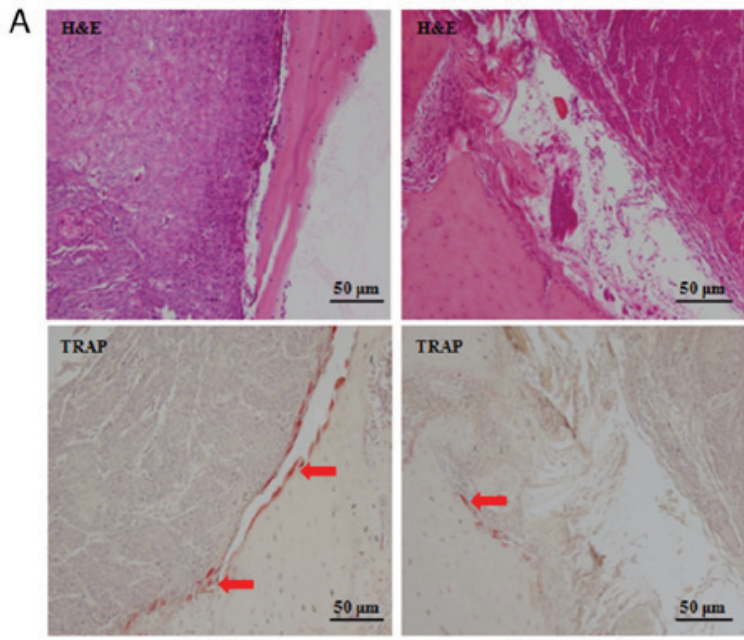

SCC25+7ND

B

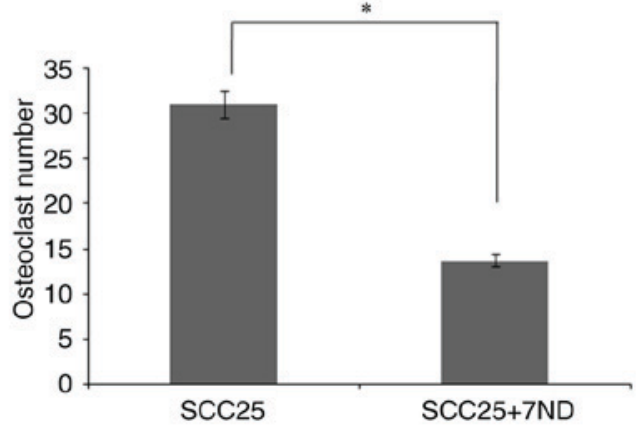

Figure 10. The number of osteoclasts at the tumor-bone interface. (A) H\&E staining of the two groups demonstrated that osteoclasts had accumulated at the tumor-bone interface. TRAP staining suggested significantly fewer osteoclasts localized to the resorption lacunae upon 7ND protein treatment. (B) Results of quantification. Data are presented as the mean \pm the standard error of the mean of 6 mice for each group. Arrow, 3,3'-diaminobenzidine; scale bar $=25 \mu \mathrm{m}$; ${ }^{*}<0.05$. TRAP, tartrate-resistant acid phosphatase. 
research design and manuscript revising; JQ contributed to the work of animal model establishment and research design.

\section{Ethics approval and consent to participate}

Written informed consent was obtained from all patients and healthy donors enrolled in the present study. All protocols were reviewed and approved by the University Ethics Committee of Sun Yat-Sen University (2016-334QX).

\section{Consent for publication}

All participants of the study provided consent for the data to be published.

\section{Competing interests}

The authors declare that they have no competing interests.

\section{References}

1. Lehmann MH, Torres-Domínguez LE, Price PJ, Brandmüller C, Kirschning CJ and Sutter G: CCL2 expression is mediated by type I IFN receptor and recruits NK and T cells to the lung during MVA infection. J Leukoc Biol 99: 1057-1064, 2016.

2. Qidwai T: Chemokine genetic polymorphism in human health and disease. Immunol Lett 176: 128-138, 2016.

3. Kim MS, Day CJ and Morrison NA: MCP-1 is induced by receptor activator of nuclear factor- $\{$ kappa $\} \mathrm{B}$ ligand, promotes human osteoclast fusion, and rescues granulocyte macrophage colony-stimulating factor suppression of osteoclast formation. J Biol Chem 280: 16163-16169, 2005.

4. Kim MS, Day CJ, Selinger CI, Magno CL, Stephens SR and Morrison NA: MCP-1-induced human osteoclast-like cells are tartrate-resistant acid phosphatase, NFATc1, and calcitonin receptor-positive but require receptor activator of NFkappaB ligand for bone resorption. J Biol Chem 281: 1274-1285, 2006.

5. Morrison NA, Day CJ and Nicholson GC: Dominant negative MCP-1 blocks human osteoclast differentiation. J Cell Biochem 115: 303-312, 2014.

6. Quan J, Johnson NW, Zhou G, Parsons PG, Boyle GM and Gao J: Potential molecular targets for inhibiting bone invasion by oral squamous cell carcinoma: A review of mechanisms. Cancer Metastasis Rev 31: 209-219, 2012.

7. Quan J, Elhousiny M, Johnson NW and Gao J: Transforming growth factor- $\beta 1$ treatment of oral cancer induces epithelial-mesenchymal transition and promotes bone invasion via enhanced activity of osteoclasts. Clin Exp Metastasis 30: 659-670, 2013

8. Quan J, Morrison NA, Johnson NW and Gao J: MCP-1 as a potential target to inhibit the bone invasion by oral squamous cell carcinoma. J Cell Biochem 115: 1787-1798, 2014.

9. Gu J, Feng Y, Feng X, Sun C, Lei L, Ding W, Niu F, Jiao L, Yang M, Li Y, et al: Structural and biochemical characterization reveals LysGH15 as an unprecedented 'EF-hand-like' calcium-binding phage lysine. PLoS Pathog 10: e1004109, 2014.
10. Gong QM, Quan JJ, Jiang HW and Ling JQ: Regulation of the stromal cell-derived factor-1alpha-CXCR4 axis in human dental pulp cells. J Endod 36: 1499-1503, 2010.

11. Zhang Y, Ernst CA and Rollins BJ: MCP-1: Structure/activity analysis. Methods 10: 93-103, 1996.

12. Zhang Y and Rollins BJ: A dominant negative inhibitor indicates that monocyte chemoattractant protein 1 functions as a dimer. Mol Cell Biol 15: 4851-4855, 1995.

13. Ikeda Y, Yonemitsu Y, Kataoka C, Kitamoto S, Yamaoka T, Nishida K, Takeshita A,Egashira K and Sueishi K: Anti-monocyte chemoattractant protein-1 gene therapy attenuates pulmonary hypertension in rats. Am J Physiol Heart Circ Physiol 283: H2021-H2028, 2002.

14. Ni W, Egashira K, Kitamoto S, Kataoka C, Koyanagi M, Inoue S, Imaizumi K, Akiyama C, Nishida KI and Takeshita A: New anti-monocyte chemoattractant protein-1 gene therapy attenuates atherosclerosis in apolipoprotein E-knockout mice. Circulation 103: 2096-2101, 2001.

15. Koga M, Kai H, Egami K, Murohara T, Ikeda A, Yasuoka S, Egashira K, Matsuishi T, Kai M, Kataoka Y, et al: Mutant MCP-1 therapy inhibits tumor angiogenesis and growth of malignant melanoma in mice. Biochem Biophys Res Commun 365: 279-284, 2008

16. Yao Z, Keeney M, Lin TH, Pajarinen J, Barcay K, Waters H, Egashira K, Yang F and Goodman S: Mutant monocyte chemoattractant protein 1 protein attenuates migration of and inflammatory cytokine release by macrophages exposed to orthopedic implant wear particles. J Biomed Mater Res A 102: 3291-3297, 2014.

17. Jiang X, Sato T, Yao Z, Keeney M, Pajarinen J, Lin TH, Loi F, Egashira K, Goodman S and Yang F: Local delivery of mutant CCL2 protein-reduced orthopaedic implant wear particle-induced osteolysis and inflammation in vivo. J Orthop Res 34: 58-64, 2016

18. Keeney M, Waters H, Barcay K, Jiang X, Yao Z, Pajarinen J, Egashira K, Goodman SB and Yang F: Mutant MCP-1 protein delivery from layer-by-layer coatings on orthopedic implants to modulate inflammatory response. Biomaterials 34: 10287-10295, 2013.

19. Nabeshima A, Pajarinen J,Lin TH, Jiang X, Gibon E, Córdova LA, Loi F, Lu L, Jämsen E, Egashira K, et al: Mutant CCL2 protein coating mitigates wear particle-induced bone loss in a murine continuous polyethylene infusion model. Biomaterials 117: 1-9, 2017.

20. Severin IC, Souza AL, Davis JH, Musolino N, Mack M, Power CA and Proudfoot AE: Properties of 7ND-CCL2 are modulated upon fusion to Fc. Protein Eng Des Sel 25: 213-222, 2012.

21. Russmueller G, Moser D, Würger T, Wrba F, Christopoulos P, Kostakis G, Seemann R, Stadler V, Wimmer G, Kornek G, et al: Upregulation of osteoprotegerin expression correlates with bone invasion and predicts poor clinical outcome in oral cancer. Oral Oncol 51: 247-253, 2015.

22. Tada Y, Kokabu S, Sugiyama G, Nakatomi C, Aoki K, Fukushima H, Osawa K, Sugamori Y, Ohya K, Okamoto M, et al: The novel IкB kinase $\beta$ inhibitor IMD- 0560 prevents bone invasion by oral squamous cellcarcinoma. Oncotarget 5: 12317-12330, 2014.

23. Hsu FT, Chang B, Chen JC, Chiang IT, Liu YC, Kwang WK and Hwang JJ: Synergistic effect of sorafenib and radiation on human oral carcinoma in vivo. Sci Rep 5: 15391, 2015.

This work is licensed under a Creative Commons Attribution-NonCommercial-NoDerivatives 4.0 International (CC BY-NC-ND 4.0) License. 